\title{
Commentary
}

\section{New Look at an Old Problem}

\section{Bacterial Superinfection after Influenza}

\author{
Kevan L. Hartshorn \\ From the Department of Medicine, Boston University School of \\ Medicine, Boston, Massachusetts
}

Influenza A viruses (IAV) continue to be an important cause of morbidity and mortality worldwide. The ability of IAV to acquire small mutations because of the infidelity of the viral RNA polymerase (resulting in antigenic drift) or major changes resulting from introduction of whole RNA genome segments from animal IAV strains (antigenic shift) results in frequent introduction of new strains that, to greater or lesser degrees, evade adaptive immune responses developed against prior strains. A complex and largely effective innate immune response allows humans to contain IAV replication during the first several days after infection, pending the development of adaptive immune responses (see White et al ${ }^{1}$ for review). Indeed, it is now increasingly accepted that mortality from IAV is not often attributable to viral pneumonia per se (although this does occur and is potentially lethal), but rather that IAV predisposes to virulent bacterial infections, which account for much of the mortality during IAV epidemics and pandemics.

The 1918 IAV pandemic caused massive morbidity and mortality ( $\approx 50$ million deaths worldwide). Reconstruction of the $1918 \mathrm{H} 1 \mathrm{~N} 1$ strains confirmed that the virus itself was highly pathogenic; however, recent reviews of available epidemiological and pathological data demonstrate that bacterial pneumonias accounted for the majority of deaths in that pandemic. ${ }^{2,3}$ The most common bacterial agent found in post-IAV pneumonias in 1918, and subsequently, is Streptococcus pneumoniae (S. pneumoniae) ${ }^{2}$; however, an association of IAV infection with staphylococcal pneumonia has been repeatedly reported, including alarming recent reports of pneumonia and mortality attributable to methicillin-resistant staphylococcal infections in young subjects with IAV infection. ${ }^{4}$ Moreover, superinfection with Haemophilus influenzae ( $H$. influenzae) was especially prominent during the 1918 IAV pandemic, although it has been reported in subsequent epidemics and pandemics as well. ${ }^{2}$ In addition to predisposing to bacterial pneumonia, IAV infection promotes other bacterial infections that enter through a respiratory route, including meningitis and otitis media. $^{5}$

A major concern in treating post-IAV infections is that the mortality rate from bacterial pneumonia occurring in IAV-infected subjects remains high despite use of antibiotics. ${ }^{6}$ Another striking feature of post-IAV bacterial infections is their occurrence and mortality rate among otherwise young and healthy individuals. ${ }^{2,4} \mathrm{~A}$ high rate of bacterial superinfection has now been identified in children who have died from the novel $\mathrm{H} 1 \mathrm{~N} 1$ virus that is currently causing a pandemic. ${ }^{7}$ It is, therefore, critical that we reach a better understanding of the causes and potential treatments for post-IAV bacterial superinfection.

The article by Lee et al in this volume of The American Journal of Pathology provides a valuable addition to the literature on post-IAV bacterial superinfection. ${ }^{8}$ There are now well-characterized models that have begun to elucidate the mechanisms of post-IAV pneumococcal infections (for an excellent review see McCullers ${ }^{9}$ ); however, relatively little literature is available relating to animal models of other common bacteria that complicate IAV infection, including Staphylococcus aureus (S. aureus) and $H$. influenzae. Lee et al provide a very well-characterized mouse model confirming that otherwise nonlethal IAV or $\mathrm{H}$. influenzae infections cause high mortality rates in mice when IAV infection precedes inoculation with $H$. influenzae. The study clearly demonstrates the time period of most vulnerability to $\mathrm{H}$. influenzae-induced mortality ( 3 to 4 days post-IAV infection), the viral and bacterial dose dependency for mortality, and the fact that the major problem is a strong potentiation of bacterial (and not viral) growth and its associated pathological effects after IAV infection. A number of early studies, performed in the 1930's and 1940's (ie, soon after the first isolation of $I A V)$, demonstrated increased mortality in pigs, guinea pigs, and mice after co-infection with IAV and $H$. influen-

Supported by NIH grants HL069031 and AI083222.

Accepted for publication October 5, 2009

Address reprint requests to Kevan L. Hartshorn, M.D., Boston University School of Medicine, EBRC 414, 650 Albany Street, Boston, MA 02118 E-mail: khartsho@bu.edu. 
zae (see Lee et al ${ }^{8}$ for summary), and the current study by Lee et $\mathrm{al}^{8}$ confirms and extends on these findings. This very well characterized model provides the foundation for studies of mechanisms through which IAV impairs antibacterial defense (see below).

\section{Current Understanding of Etiology of Bacterial Superinfections Post-IAV}

A rich literature has recently emerged regarding the possible ways in which IAV paves the way for pneumococcal superinfection. It is now clear that IAV causes defects in innate immunity to pneumococcal infection of the respiratory tract. The study by Lee et al and other studies involving animal models of $S$. aureus infection post-IAV ${ }^{10}$ are valuable in that they indicate that the IAV-induced defect is probably broad-based and affects immunity to a variety of bacteria (as one would expect from the clinical data). There is evidence of a role for specific bacterial and viral components in mediating bacterial superinfection that will first be summarized, followed by the recent findings regarding more broad-based effects of IAV on innate and adaptive immune responses.

\section{Role of Specific Bacterial Components in Superinfection}

There is evidence that specific features of individual types of bacteria contribute to superinfection. For instance, S. aureus enterotoxin (a superantigen) was shown to induce a profound lethal inflammatory response involving tumor necrosis factor- $\alpha$, interferon (IFN)- $\gamma$, and cytotoxic $T$ cells when administered 7 days after IAV infection. ${ }^{10}$ In addition, a $S$. aureus protease can potentiate $\mathrm{IAV}$ infection by promoting cleavage of the viral hemagglutinin (a prerequisite for viral infectivity). ${ }^{11}$ Similarly, protein A of $S$. pneumoniae (and not the bacterial neuraminidase or hyaluronidase) has been found to contribute to post-IAV superinfection with this bacterial species.

\section{Role of Specific Viral Components in Bacterial Superinfection}

There is also evidence that specific viral components contribute to bacterial superinfection. An obvious means through which IAV could promote bacterial infection is by increasing bacterial adhesion to airway epithelial cells. The virus has two envelope proteins that interact with target respiratory epithelial cell membrane components: neuraminidase (NA) and hemagglutinin. There is clear evidence that the action of the viral NA facilitates adhesion of S.pneumoniae to respiratory epithelial cells. The IAV NA also increases platelet activating receptor expression (which is a specific receptor for S.pneumoniae) however, this receptor was shown not to be required for increased bacterial adherence or mortality attributable to S. pneumoniae post-IAV infection. ${ }^{12}$
Importantly NA inhibition using oseltamivir has been shown to inhibit mortality resulting from $S$. pneumoniae superinfection post-IAV in mice. ${ }^{6}$ The amantadines, which block the viral $\mathrm{M}$ protein that serves as a proton channel, did not have this effect. These findings are of interest because clinical trials of oseltamivir show reduction in bacterial complications of IAV infection, whereas similar results have not been reported with the adamantanes. ${ }^{6}$

Viral hemagglutinin also has been shown to inhibit neutrophil responses. ${ }^{13}$ IAV causes deactivation of neutrophil functions (like chemotaxis, respiratory burst, degranulation, and bacterial killing) in vitro and in vivo, and this could contribute to impaired containment of bacteria at times when viral replication is still active. ${ }^{5}$

There are two IAV proteins that appear to function primarily to interfere with host immune responses, the NS1 and PB1-F2 proteins. NS1 inhibits various aspects of the innate antiviral response, including type I IFN response pathways, and the PB1-F2 protein (a product of an alternative reading frame of one of the polymerase genes of IAV) induces apoptosis of host immune cells. The specific contribution of the NS1 protein to bacterial superinfection has not been reported; however, deletion of the PB1-F2 protein from the PR-8 strain of IAV reduced mortality from S. pneumoniae superinfection. ${ }^{14}$ The presence of the PB1-F2 protein promoted bacterial growth (as assessed using luminescent bacteria and whole mouse imaging) and markedly potentiated inflammatory responses to infection (including influx of neutrophils and macrophages into the lung). Insertion of the PB1-F2 protein from a 1918 pandemic IAV strain into the PR-8 viral strain caused increased mortality from post-IAV bacterial infection compared with the wild-type PR-8 virus (containing its own version of PB1-F2). These findings may in part account for the high mortality of the 1918 pandemic.

\section{Mechanisms to Account for a Broad-Based Defect in Antibacterial Defense Caused by IAV Infection}

One obvious potential mechanism for the increased incidence of bacterial pneumonias in IAV-infected subjects is that there are common risk factors for both types of infection. For instance, individuals with diabetes mellitus, chronic obstructive pulmonary disease, smoking history, or immuno-compromised states are at risk for both types of infection. This seems unlikely to account for the tight association seen between waves of IAV infection and subsequent pneumonia, especially given that this association is observed often in otherwise healthy individuals. Another explanation that has greater merit is that IAV damages the respiratory mucosa and the ciliary apparatus, interfering with natural barriers to bacterial attachment and invasion. Such a mechanism could be shared by other viruses and, indeed, respiratory syncytial virus, metapneumovirus, and other respiratory viruses also predispose to bacterial superinfection. ${ }^{15,16}$ This mechanism and possible direct interactions between viruses and 
bacteria (eg, as in the $S$. aureus protease model) would be less likely to account for the potentiation of bacterial superinfection that occurs after clearance of the virus (eg, when bacteria inoculation occurs two weeks or more after IAV infection ${ }^{17}$ ).

Recent studies have focused on innate immune responses triggered by IAV that could suppress subsequent response to bacterial infection. One example is the ability of IAV to elicit a strong interleukin (IL)-10 response in a delayed manner after infection. Inhibition of this IL-10 response was protective against $S$. pneumoniae superinfection $^{18}$; however, because studies with IL-10 knockout mice did not support these findings, ${ }^{19}$ the role of IL-10 in bacterial superinfection is controversial. Two recent studies have shown a role for IAV-induced IFN generation in bacterial superinfection. In one study IFN- $\gamma$ triggered by IAV was found to depress macrophage function (including reducing expression of the macrophage scavenger receptor, MARCO) leading to impaired early phases of S. pneumoniae clearance. ${ }^{19} \mathrm{IFN}-\gamma$ knockout mice were relatively protected from $S$. pneumoniae-induced mortality. In another study, type I IFNs (ie, IFNs $\alpha$ and $\beta$ ) elicited by IAV were strongly implicated in predisposing mice to mortality from S. pneumoniae superinfection. ${ }^{20}$ A prior study had shown that type I IFN induction by lymphocytic choriomeningitis virus results in profound depression of neutrophil responses and increased susceptibility to bacterial infection. ${ }^{21}$ Similarly, IAV infection was found to induce type I IFN generation resulting in depression of neutrophil response to subsequent respiratory $S$. pneumoniae infection. ${ }^{20}$ This was shown using mice lacking the common receptor for type I IFNs (Ifnar ${ }^{-1-}$ mice) and was specifically related to impaired macrophage inflammatory protein and Keratinocyte-derived chemokine generation in these mice. Macrophage inflammatory protein and Keratinocyte-derived chemokine are chemokines responsible for neutrophil recruitment to the lung in mice (similar to IL-8 in humans). Treatment with macrophage inflammatory protein or Keratinocyte-derived chemokine was protective against bacterial superinfection in IAVinfected wild-type mice, suggesting a possible future therapeutic approach. The authors of this article, ${ }^{20}$ and the article showing a role for IFN- $\gamma,{ }^{19}$ conclude that innate responses geared to increase host protection against IAV may have the unfortunate consequence of predisposing people to subsequent bacterial infection.

IFN- $\gamma$ has been found to be unnecessary for successful clearance of IAV in mice; however, type I IFNs are more important for defense against IAV (in part depending on the model system being used). It will be of interest to determine the effects of IFN- $\lambda$ on bacterial defense, because this has been recently found to be strongly triggered by IAV in respiratory epithelial cells. ${ }^{22}$ The findings with IFNs appear to be consistent with findings of another important recent study, which showed that IAV infection of mice causes a prolonged down-regulation of several toll-like receptors in macrophages that correlates with susceptibility to bacterial superinfection. ${ }^{17}$ In this model chemokine generation and neutrophil recruitment were also impaired.

\section{Placing the Findings of Lee et al in the Context of Recent Literature}

The findings of Lee et al need to be viewed in the context of the recent literature regarding mechanisms of bacterial superinfection. Lee et al make heroic efforts to identify specific adaptive or innate immune mediators triggered by IAV that could promote bacterial infection. These studies involve application of their well-characterized $\mathrm{H}$. influenzae superinfection model to a variety of knockout mice, involving both adaptive and innate response elements. Recruitment of CD8 T cells specific for IAV takes five or more days after initial infection of a naïve host, and they are necessary for ultimate clearance of IAV. Lee et al evaluate the role of CD8 T cells attributable to the coincidence in timing between expected arrival of these cells and peak mortality from bacterial superinfection. In some models excessive induction of CD8 T cell-mediated cytotoxicity has been associated with IAV-induced mortality; however, Lee et al found no major alteration in the rate and timing of mortality after $\mathrm{H}$. influenzae superinfection in mice lacking fas, CXCR3, or recombinanase activating gene (RAG). Fas antigen is an important mechanism through which CD8 $T$ cells mediate apoptosis of target cells, CXCR3 is involved in recruitment of T and NK cells to the lung, and RAG is required for gene recombination necessary for adaptive immunity. Although the RAG ${ }^{-1-}$ mice suffered mortality from IAV infection alone, the timing of mortality was greatly accelerated by bacterial superinfection. Hence, these results appear to exclude a contribution of harmful adaptive immune responses to bacterial superinfection.

Some studies have found that excessive innate inflammatory responses, rather than excessive viral replication per se, are responsible for IAV-associated mortality. ${ }^{1}$ For instance, recruitment of activated macrophages through CCR2, excessive induction of reactive oxygen and nitrogen species, or stimulation of acute lung injury through TLR4 receptors have been found to be injurious in various mouse models of severe IAV infection. ${ }^{1}$ It should be noted that these models differ from the typical bacterial superinfection models in that higher lethal doses of virus, or more pathogenic viral strains (eg, H5N1), are used in the former studies. In any case, Lee et al did not find that $\mathrm{CCR}^{-1-}$ mice or mice lacking functional TLR4 (ScNJ mice) had appreciably different mortality after bacterial superinfection. In addition, tumor necrosis factor- $\alpha$ and IL-6 are strongly stimulated by IAV infection and play some role in virus-induced symptomatology and antiviral defense. Although mice lacking these cytokines had some increase in mortality from viral infection alone, mortality was more rapid and complete (and paralleled that of wild-type mice closely) after bacterial superinfection.

The efforts of Lee et al did not, therefore, identify specific causes of mortality in $\mathrm{H}$. influenzae-superinfected mice despite partially excluding a number of important possible immune mediators. It should also be noted that it is not possible to formally exclude a role for all of the immune response elements studied by Lee et al because some of the knockouts (eg, of RAG, tumor necrosis factor 
R, or IL-6) affect resistance to IAV per se so that lower viral inocula had to be used. An important question for future study will be whether IFNs play a role in IAVinduced susceptibility to $H$. influenzae or other bacterial infections (in addition to S. pneumoniae infection). Lee et al also did not evaluate the role of specific viral or bacterial components in superinfection, and this can also be addressed in the future.

\section{Conclusions}

The observation that bacterial superinfections complicate influenza dates back at least to the early 1800's when Laennec noted increased incidence of pneumonias after an influenza epidemic. ${ }^{9}$ Clearly such bacterial superinfections are a major cause of morbidity and mortality worldwide to this day, despite widespread availability of antibiotics. Based on recent studies, including those of Lee et al in this volume, an understanding of the pathogenesis of post-IAV bacterial infections is now taking shape. It is clear that IAV induces defects in respiratory mucosal immunity that are broad-based and adversely affect response to a range of bacteria. The defects mainly appear to involve innate (rather than adaptive) immune barriers to bacterial adhesion and invasion, including alterations in the mucosa itself and adverse consequences of early innate mediators, like IFNs, which are involved in the antiviral response. The implications of these findings for treatment are not yet fully established.

The most important intervention for post-IAV bacterial infections still remains effective vaccination for influenza, because this reduces both viral infection and mortality from bacterial pneumonias. ${ }^{23}$ There is also evidence that combined treatment with NA inhibitors and antibiotics may be indicated, ${ }^{6}$ and that antibiotics that inhibit protein synthesis (like clindamycin or azithromycin) are more advantageous because of their anti-inflammatory effects. ${ }^{24}$ Another approach to treatment could involve use of broad spectrum innate inhibitors, like collectins or defensins, which inhibit both IAV and bacteria. ${ }^{1}$ The model developed by Lee et al should be useful to test such treatment strategies.

\section{References}

1. White M, Doss M, Boland P, Tecle T, Hartshorn K: Innate immunity to influenza virus: implications for future therapy. Expert Rev Clin Immunol 2008, 4:497-514

2. Morens DM, Taubenberger JK, Fauci AS: Predominant role of bacterial pneumonia as a cause of death in pandemic influenza: implications for pandemic influenza preparedness. J Infect Dis 2008, 198:962-970

3. Brundage JF, Shanks GD: Deaths from bacterial pneumonia during 1918-19 influenza pandemic. Emerg Infect Dis 2008, 14:1193-1199

4. Kallen AJ, Brunkard J, Moore Z, Budge P, Arnold KE, Fosheim G, Finelli L, Beekmann SE, Polgreen PM, Gorwitz R, Hageman J: Staphylococcus aureus community-acquired pneumonia during the 2006 to 2007 influenza season. Ann Emerg Med 2008
5. Hartshorn K: Etiology of Bacterial Superinfections complicating influenza viral infection. Options for the Control of Influenza. Edited by Brown L, Hampson A, Webster R. Amsterdam, Elsevier Science, 1996, pp 499-508

6. McCullers JA: Effect of antiviral treatment on the outcome of secondary bacterial pneumonia after influenza. J Infect Dis 2004, 190:519-526

7. Surveillance for pediatric deaths associated with 2009 pandemic influenza A (H1N1) virus infection-United States, April-August 2009 MMWR Morb Mortal Wkly Rep 2009, 58:941-947

8. Lee L, Dias P, Han D, Yoon S, Zakharov V, Parham D, Sarawar S: A mouse model of lethal synergism between influenza virus and Haemophilus influenzae. Am J Pathol 2010, 176:800-811

9. McCullers JA: Insights into the interaction between influenza virus and pneumococcus. Clin Microbiol Rev 2006, 19:571-582

10. Zhang WJ, Sarawar S, Nguyen P, Daly K, Rehg JE, Doherty PC, Woodland DL, Blackman MA: Lethal synergism between influenza infection and staphylococcal enterotoxin B in mice. J Immunol 1996, 157:5049-5060

11. Tashiro M, Ciborowski P, Klenk H, Pulverer G, Rott R: Role of Staphyloccus protease in the development of influenza pneumonia. Nature 1987, 325:536-537

12. McCullers JA, Iverson AR, McKeon R, Murray PJ: The platelet activating factor receptor is not required for exacerbation of bacteria pneumonia following influenza. Scand J Infect Dis 2008, 40:11-17

13. Hartshorn KL, Liou LS, White MR, Kazhdan MM, Tauber JL, Tauber Al: Neutrophil deactivation by influenza A virus. Role of hemagglutinin binding to specific sialic acid-bearing cellular proteins. J Immunol 1995, 154:3952-3960

14. McAuley JL, Hornung F, Boyd KL, Smith AM, McKeon R, Bennink J, Yewdell JW, McCullers JA: Expression of the 1918 influenza A virus PB1-F2 enhances the pathogenesis of viral and secondary bacterial pneumonia. Cell Host Microbe 2007, 2:240-249

15. Johnstone J, Majumdar SR, Fox JD, Marrie TJ: Viral infection in adults hospitalized with community-acquired pneumonia: prevalence, pathogens, and presentation. Chest 2008, 134:1141-1148

16. Kukavica-Ibrulj I, Hamelin ME, Prince GA, Gagnon C, Bergeron Y, Bergeron MG, Boivin G: Infection with human metapneumovirus predisposes mice to severe pneumococcal pneumonia. J Virol 2009, 83:1341-1349

17. Didierlaurent A, Goulding J, Patel S, Snelgrove R, Low L, Bebien M, Lawrence T, van Rijt LS, Lambrecht BN, Sirard JC, Hussell T: Sustained desensitization to bacterial Toll-like receptor ligands after resolution of respiratory influenza infection. J Exp Med 2008, 205:323-329

18. Van Der Sluijs KF, Van Elden LJ, Nijhuis M, Schuurman R, Pater JM, Florquin S, Goldman M, Jansen HM, Lutter R, Van Der Poll T: IL-10 is an important mediator of the enhanced susceptibility to pneumococcal pneumonia after influenza infection. J Immunol 2004, 172:7603-7609

19. Sun K, Metzger DW: Inhibition of pulmonary antibacterial defense by interferon-gamma during recovery from influenza infection. Nat Med 2008, 14:558-564

20. Shahangian A, Chow EK, Tian X, Kang JR, Ghaffari A, Liu SY, Belperio JA, Cheng G, Deng JC: Type I IFNs mediate development of postinfluenza bacterial pneumonia in mice. J Clin Invest 2009, 119:1910-1920

21. Navarini AA, Recher M, Lang KS, Georgiev P, Meury S, Bergthaler A Flatz L, Bille J, Landmann R, Odermatt B, Hengartner $\mathrm{H}$, Zinkernagel RM: Increased susceptibility to bacterial superinfection as a consequence of innate antiviral responses. Proc Natl Acad Sci USA 2006, 103:15535-15539

22. Wang J, Oberley-Deegan R, Wang S, Nikrad M, Funk CJ, Hartshorn $\mathrm{KL}$, Mason RJ: Differentiated human alveolar type II cells secrete antiviral IL-29 (IFN-lambda1) in response to influenza A infection. J Immunol 2009, 182:1296-1304

23. Kandel R, Hartshorn KL: Novel strategies for prevention and treatment of influenza. Expert Opin Ther Targets 2005, 9:1-22

24. Karlstrom A, Boyd KL, English BK, McCullers JA: Treatment with protein synthesis inhibitors improves outcomes of secondary bacterial pneumonia after influenza. J Infect Dis 2009, 199:311-319 